\title{
Aplicación del análisis de redes al estudio de la investigación española de historia de la ciencia
}

\author{
Julia Osca-Lluch ${ }^{1}$ \\ Instituto de Historia de la Medicina y de la Ciencia López Piñero (UV-CSIC)
}

\section{Resumen}

El presente trabajo analiza los patrones de colaboración en España, de los investigadores en historia de la ciencia, a través de un análisis de coautoría en las publicaciones científicas del área, recogidas en las bases de datos Social Science Citation Index (SSCl) y Science Citation Index (SCl) durante el período 1990-2009. Los resultados permiten detectar un aumento de la producción científica a lo largo del periodo y una escasa colaboración científica, ya que solamente el $18,61 \%$ del total de los trabajos han sido firmados por más de un autor.

Palabras clave: Redes sociales, Análisis de redes sociales, Bibliometría, Historia de la ciencia, España

\begin{abstract}
This paper analyzes the patterns of cooperation in Spain researchers in history of science, through an analysis of co-authorship in scientific publications in the area, contained in the databases Social Science Citation Index (SSCI) and Science Citation Index $(\mathrm{SCl})$ for the period 1990-2009. The results show an increase in scientific output over a limited period and scientific collaboration, as only $18,61 \%$ of the papers have been signed by more than one author.
\end{abstract}

Key words: Social networks, Social networks analysis, Bibliometrics, History of Science, Spain

\footnotetext{
${ }^{1}$ Enviar correspondencia a: m.julia.osca@uv.es
} 


\section{I ntroducción}

Una parte esencial de la investigación la constituye la publicación científica. La labor científica se suele medir a través de las publicaciones y otros resultados que los científicos producen. Machlup (1962) afirmaba que el conocimiento nuevo no se crea hasta que ha sido transmitido a otros, y entre los posibles modos de transmisión, sin duda es la publicación científica el más importante porque además de fijar el conocimiento permite su contrastación y contestación ya que los procesos del sistema científico solo llegan a su culminación cuando se hacen públicos.

Entre los métodos más utilizados para evaluar distintos aspectos de la actividad investigadora hay que destacar el papel fundamental de los análisis bibliométricos de la producción científica, junto con los tradicionales indicadores de input como el número de investigadores, los recursos destinados a I+D+I o el número de equipos disponibles. Los estudios bibliométricos se han considerado de interés para el análisis de aspectos fundamentalmente cuantitativos de la actividad científica, así como para el estudio de su evolución temporal y su utilización permite una aproximación objetiva a una realidad compleja y multifacética como es la investigadora.

Los estudios bibliométricos, basados en datos procedentes de las publicaciones científicas, han experimentado un gran desarrollo en las dos últimas décadas tanto en España como en el resto de los países avanzados. El interés de este tipo de estudios radica en que permiten obtener una visión general de la actividad científica de un país, centro o área, y realizar comparaciones y seguimientos a lo largo del tiempo. Entre los demandantes de estos análisis se sitúan los propios investigadores, deseosos de conocer el "mapa" de la actividad investigadora que se realiza en su área científica, pero también los gestores de política científica, a los que proporcionan datos objetivos de apoyo en su toma de decisiones (Zulueta et al., 1999).

Durante los últimos años, la aplicación de los métodos y el estudio sobre las diferentes redes sociales han pasado de ser un instrumento empleado únicamente por un núcleo muy reducido de especialistas en estudios sociales sobre la ciencia (López Piñero, 1972) a convertirse en un instrumento cada día más utilizado por los especialistas en documentación, que pueden aplicar el análisis de redes complementariamente a las técnicas bibliométricas utilizadas para el estudio sobre producción científica en los ámbitos de coautoría y colaboración de autores, instituciones y pautas de citación (Molina, 2001; Molina et al., 2002; Delgado et al., 2006). 
No obstante, como es ampliamente conocido, no todas las publicaciones tienen el mismo valor para los científicos, ni los hábitos de publicación y difusión son los mismos en las diferentes áreas o disciplinas científicas. En las ciencias experimentales la información se transmite principalmente a través de artículos de revista, por el contrario, en las ciencias sociales y las humanidades y, por distintos motivos, también en las aplicaciones prácticas, la información científica se transmite principalmente a través de los libros, en tanto que los artículos de revistas tienen un peso relativamente modesto (Terrada et al., 1993).

Tampoco hay que olvidar que los indicadores bibliométricos, presentan algunas limitaciones que hay que tener presente a la hora de su utilización (López Piñero y Terrada, 1992; Camí, 1997; Osca-Lluch et al., 2003; Alfonso et al., 2005). Así por ejemplo, una cantidad de conocimiento no se puede medir, dado que no se publica por los cauces y sistemas habituales de comunicación formal. Además, parte de los resultados de la investigación aplicada y, de forma mayoritaria los del desarrollo experimental, no se publican en revistas científicas, sino en otro tipo de documentos (patentes, informes técnicos, actas de congresos, etc.).

Las dificultades de utilización de este tipo de estudios en todos los niveles (macro, meso, micro) para delimitar la posición del sistema de ciencia han sido ampliamente comentados en la literatura de la especialidad y los resultados de investigación se presentan bien como medición de las capacidades productivas o bien como medición de la visibilidad a nivel internacional (Fecyt, 2006). Los estudios bibliométricos han ido ganando popularidad debido a su complementariedad con la economía, el análisis estructural de redes sociales, etc., y se fundamentan en una serie de premisas y limitaciones que se basan en la noción de que la esencia de la investigación científica es la comunicación de nuevas contribuciones al corpus de conocimiento de la literatura científica. La idea de publicar los resultados de la investigación es una de las actividades de los científicos y aunque existan distintos canales por los que se difundan, el corpus bibliográfico está definido en términos de bibliografía impresa.

Sin embargo, las evaluaciones de la actividad investigadora en las áreas humanas y sociales carecen habitualmente de una base cuantitativa adecuada, confiando las evoluciones a comités de expertos, que han de desarrollar criterios de evaluación ad hoc, lo que lleva implícito elementos de subjetividad en el resultado de las mismas (López Baena et al., 2005). También, a la hora de estudiar la situación de la investigación en humanidades en España es necesario tener en cuenta ciertos condicionantes preliminares que la diferencian de sus equivalentes en otros ámbitos 
científicos, como es el escaso peso relativo de las humanidades en el conjunto de la financiación pública e investigación y la práctica inexistencia de financiación privada en el sector (Fecyt, 2006).

En el caso de las Humanidades, según Cuenca (2003), una de las actuaciones fundamentales de apoyo a la investigación se debe centrar en la potenciación y mejora de las publicaciones científicas. Es importante primar la difusión de la investigación en revistas internacionales de prestigio, a fin de alcanzar el reconocimiento del trabajo realizado en España, al tiempo que se apoya a aquellas revistas nacionales que cumplan los estándares más exigentes de rigor en la selección del material publicado y se arbitran mecanismos para impulsar el establecimiento de procedimientos rigurosos de selección, con evaluación externa $y$, al menos, parcialmente, en las lenguas de referencia en el campo científico, capaces de insertarse en el panorama internacional.

La colaboración científica entre los autores y las instituciones es un rasgo diferencial de la investigación que se realiza actualmente que ha experimentado un enorme auge en las últimas décadas, hasta el punto de que los grupos de investigación han pasado a ser la mínima unidad del sistema científico en muchas de sus áreas. Cada vez es más frecuente, que los trabajos de investigación sean firmados por un número mayor de autores e instituciones. Esto, que hasta hace relativamente poco tiempo, estaba restringido a las ciencias puras, experimentales y a las ingenierías, se está extendiendo, aunque con menor intensidad a las ciencias sociales y a las humanidades.

En el análisis de las comunidades de investigación cada vez se potencia y estudia más la colaboración entre científicos del mismo u otro país, al observarse sus efectos beneficiosos en múltiples facetas de la actividad científica, desde la formación de investigadores, a la visibilidad de sus resultados (Russell et al., 2006, 2007). Es innegable que actualmente las estrategias de trabajo de los científicos poco tienen que ver con las prácticas de hace diez o veinte años. Este hecho se ha venido reflejando en el incremento de trabajos en colaboración por parte de autores de diferentes latitudes o centros de investigación y desarrollo. En la literatura sobre el tema, el incremento de la colaboración científica es explicada por diversas razones, entre ellas sobresale la necesidad de tener acceso a equipos de alta tecnología, la búsqueda por hacer más eficiente el uso de recursos financieros e incluso el interés por colaborar con especialistas renombrados (Russell et al., 2006). Según un reciente estudio realizado sobre la colaboración científica de las universidades españolas, seis de cada diez artículos publicados se escriben en 
colaboración y tres de cada diez cuentan con colaboradores internacionales (Olmeda-Goméz et al., 2009).

En la bibliometría, entendida como el conjunto de métodos utilizados en el estudio o en la medición de textos e información, se establece la coautoría de documentos científicos como una manifestación cuantificable de la colaboración entre investigadores, instituciones y países. A través del análisis de los elementos bibliográficos de autoría de los artículos, compuestos por nombres y afiliaciones institucionales, se identifican las redes de colaboración en la ciencia, desde sus distintos niveles, locales, regionales o internacionales (Russell et al., 2009) y permite establecer indicadores significativos de la estructura de influencias, al menos tal como es percibida por los propios autores (Molina et al., 2002).

El objetivo de este trabajo es el estudio de la actividad científica española en historia de la ciencia, a nivel internacional, durante el período 1990-2009, con el fin de conocer los hábitos de publicación, colaboración y difusión de la investigación que se realiza en España en historia de la ciencia a lo largo de los últimos veinte años.

Los diferentes estudios realizados sobre la coautoría o colaboración científica coinciden en afirmar que en las ciencias médicas y experimentales, así como en algunas disciplinas que se integran dentro de las ciencias sociales, como la psicología o la economía, manifiestan los más altos porcentajes de trabajos en colaboración. Desde este punto de vista, el estudio de la producción científica en historia de la ciencia, tiene gran interés por diferentes motivos, entre los que cabe señalar el carácter multidisciplinar de la propia disciplina e incluso el de los mismos científicos, ya que entre estos se observa algunas diferencias, los hay que tienen una formación académica propia de las áreas de "ciencias" (medicina, física, biología, química, etc.) y otros, sin embargo, que tienen una formación más "humanística" (historia, filosofía, etc.), lo que contribuye no solo a que exista una gran variedad en los temas objeto de estudio dentro de la propia disciplina, sino también a que existan diferencias en cuanto a los hábitos de publicación y colaboración científica entre sus profesionales.

En todo caso, hay que tener presente que este trabajo explica sólo de forma parcial la actividad científica española realizada en historia de la ciencia y que, tal como recogen algunos autores, la investigación de los científicos sociales y humanistas, no puede ser medida con precisión a partir de métodos cuantitativos, debido al carácter local y regional que tienen sus investigaciones, y que parte de esa 
bibliografía nacional, por tanto, no estará recogida en las fuentes de información internacionales.

\section{Las fuentes de información}

El estudio se ha realizado utilizando como fuente de información los trabajos sobre historia de la ciencia, realizados en alguna institución española, que han sido publicados durante el período 1990-2009 en revistas indizadas por las bases de datos multidisciplinares Social Science Citation Index ( $\mathrm{SSCl}$ ) y Science Citation Index ( $\mathrm{SCl})$. La consulta a estas dos bases de datos se ha realizado a través de la plataforma Web of Science, en el que están disponibles la versión Expanded de la base de datos Science Citation Index ( $\mathrm{SCl}$ ), y Social Science Citation Index (SSCI). Además se ha extraído información complementaria relativa a las revistas cubiertas por el ISI durante los años 1990-2009, contenidas en el Journal Citation Report (JCR) para la versión Science y Social Science.

Para delimitar el área objeto de estudio, se ha optado por seleccionar las revistas recogidas en la categoría History \& Philosophy of Science (HPS) en las dos bases de datos del Journal Citation Reports (en adelante JCR) del $\mathrm{SCl}$ y del $\mathrm{SSCl}$ durante el período 1990-2009. Se optó por considerar trabajos de historia de la ciencia todos los que han sido publicados en alguna de las revistas recogidas por las bases de datos J CR ( $\mathrm{SCl}$ y SSCl) en la categoría History \& Philosophy of Science.

La identificación en las bases de datos $\mathrm{SCl}$ y $\mathrm{SSCl}$ de los trabajos publicados, donde al menos uno de los autores firmantes perteneciese a una institución española, se llevó a cabo mediante la elaboración de una estrategia de búsqueda y recuperación sobre el campo de afiliación institucional de las bases de datos, donde se registran las direcciones de las instituciones a las que pertenecen todos los autores firmantes de los trabajos. La estrategia en cuestión debía identificar todos aquellos registros que tuvieran en dicho campo al menos una institución española y que hubieran sido publicados en alguna de las revistas clasificadas en la categoría temática History \& Philosophy of Science.

De esta forma, las estrategias de búsqueda en las bases de datos Science Citation Index $(\mathrm{SCl})$ y Socials Science Citation Index ( $\mathrm{SSCl}$ ) se han construido combinando estos parámetros: periodo temporal [1990-2009], cobertura geográfica [España] y trabajos publicados en revistas clasificadas bajo la categoría History \& Philosophy of Science en las dos bases de datos del JCR (del SCl y SSCI). 
Para cada uno de los trabajos seleccionados se ha identificado a la totalidad de los autores y se ha procedido a la unificación de las diferentes variantes de las firmas de sus nombres, con el fin de obtener información sobre la productividad científica de cada uno de ellos. Por otro lado, a partir de los trabajos firmados por varios autores se han localizado los índices de colaboración y se ha procedido a identificar los grupos de investigación. La coautoría de trabajos publicados en revistas proporciona información útil sobre las estructuras de colaboración en la comunidad científica, que configuran las redes de colaboración. En estas redes, los nodos representan autores, y los vínculos son coautorías. Dos autores están conectados si han firmado conjuntamente uno o más artículos. La estructura de tales redes revela características importantes de una determinada comunidad científica. Los indicadores bibliométricos, así como el análisis de redes sociales aplicados al análisis de la colaboración en las publicaciones científicas, permiten identificar los principales grupos y redes de trabajo que están generando una producción científica activa en un área de conocimiento, más allá de las estructuras cooperativas formales existentes, lo cual posibilita la caracterización de su actividad científica.

La comprensión de la metodología utilizada requiere asumir una serie de premisas básicas: los grupos de investigación son definidos en términos de co-autoria, es decir, aquellos autores que firman de forma conjunta un importante porcentaje de su producción, pero no se corresponden necesariamente con una determinada estructura administrativa o institucional. Las redes de colaboración se han construido a partir de los autores más productivos dentro del período estudiado y el área estudiada, pero no necesariamente coinciden con el líder real del grupo.

Para la realización de este trabajo, toda la información recogida se ha volcado en una base de datos creada en Microsoft Access para generar los ficheros de salida necesarios para la obtención de indicadores. La construcción de las redes y las representaciones gráficas se han realizado con los programas Pajek y Ucinet.

\section{La actividad científica española}

El número total de trabajos realizados en instituciones españolas, recogidos en revistas clasificadas bajo la categoría temática History \& Philosophy of Science (HPS) por las bases de datos del ISI ascendió a 569. El primer trabajo realizado en España, publicado en una revista clasificada en esta categoría temática fue en el año 1972 y desde ese año, se observa que el número de trabajos ha ido aumentando a lo largo del tiempo (Osca-Lluch et al.,2009). En la Figura 1 se muestra la evolución de la producción española en esta disciplina durante los 
últimos veinte años. Se observa un crecimiento anual, con valores positivos en todos los años, especialmente, a partir del año 2006. Los años en que se han publicado un mayor número de trabajos son los años 2008 y el 2009. Llama la atención que más del $50 \%$ de toda la producción española en historia de la ciencia, a nivel internacional, según las fuentes de información utilizadas, se ha realizado en los últimos cinco años y que solo en los dos últimos años analizados (2008 y 2009) se han publicado un $28 \%$ de toda la producción española de los últimos veinte años. Estos datos vendrían a confirmar, de alguna manera, la teoría de algunos autores que indican que los nuevos sistemas de evaluación de la calidad de la investigación esta afectando a los hábitos de publicación de los investigadores de ciencias sociales y humanas, que intentan, publicar sus trabajos en revistas recogidas en las bases de datos de la plataforma ISI Web of Knowledge, como consecuencia de los sistemas de evaluación de la actividad investigadora vigentes en nuestro país.

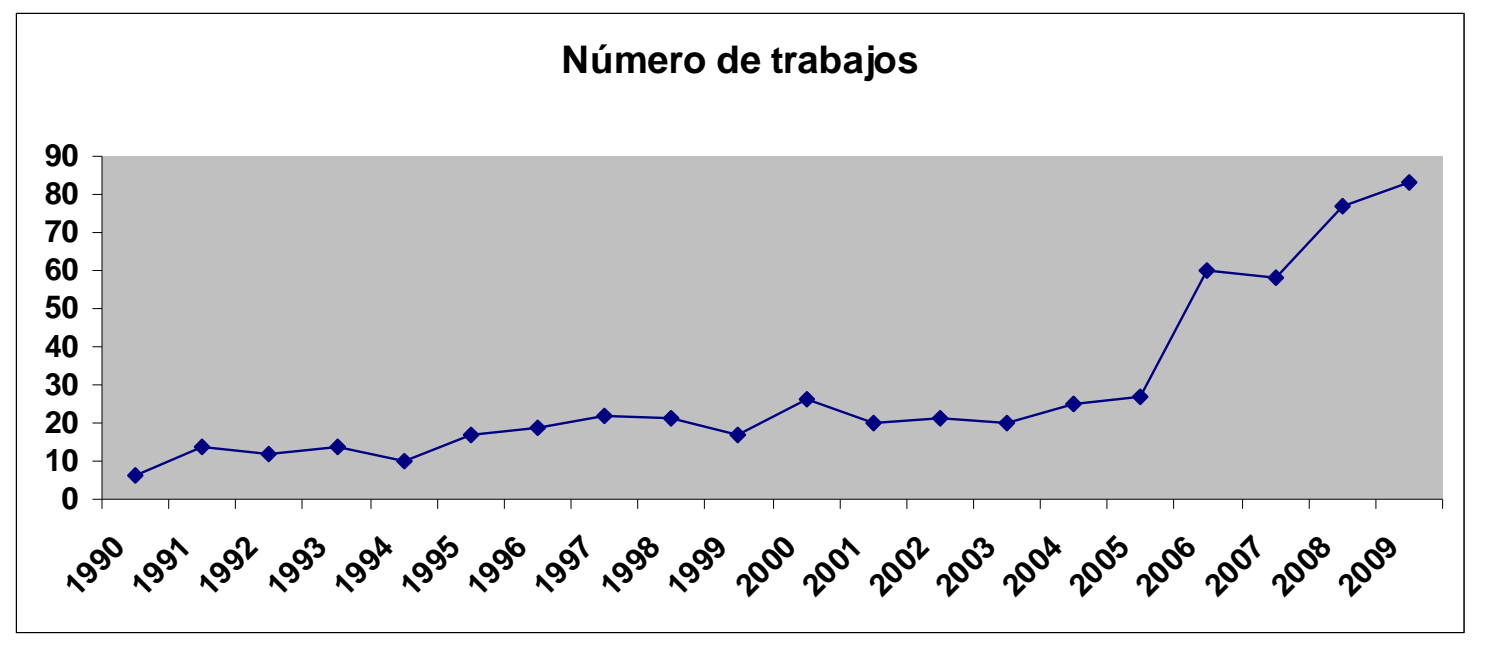

Figura 1. Evolución de la producción científica española en historia de la ciencia (1990-2009) Fuente: Elaboración propia, a partir de la base de datos $\mathrm{SCl}$ y $\mathrm{SSCl}$.

\section{Tipología documental}

La distribución de documentos por tipología documental se presenta en la Figura 2. Se observa que el mayor número de trabajos publicados corresponde a revisión de libros $(47 \%)$, artículos (39\%) y actas de congresos (7\%), quedando el $7 \%$ restante de la producción total distribuida entre otros tipos documentales. 


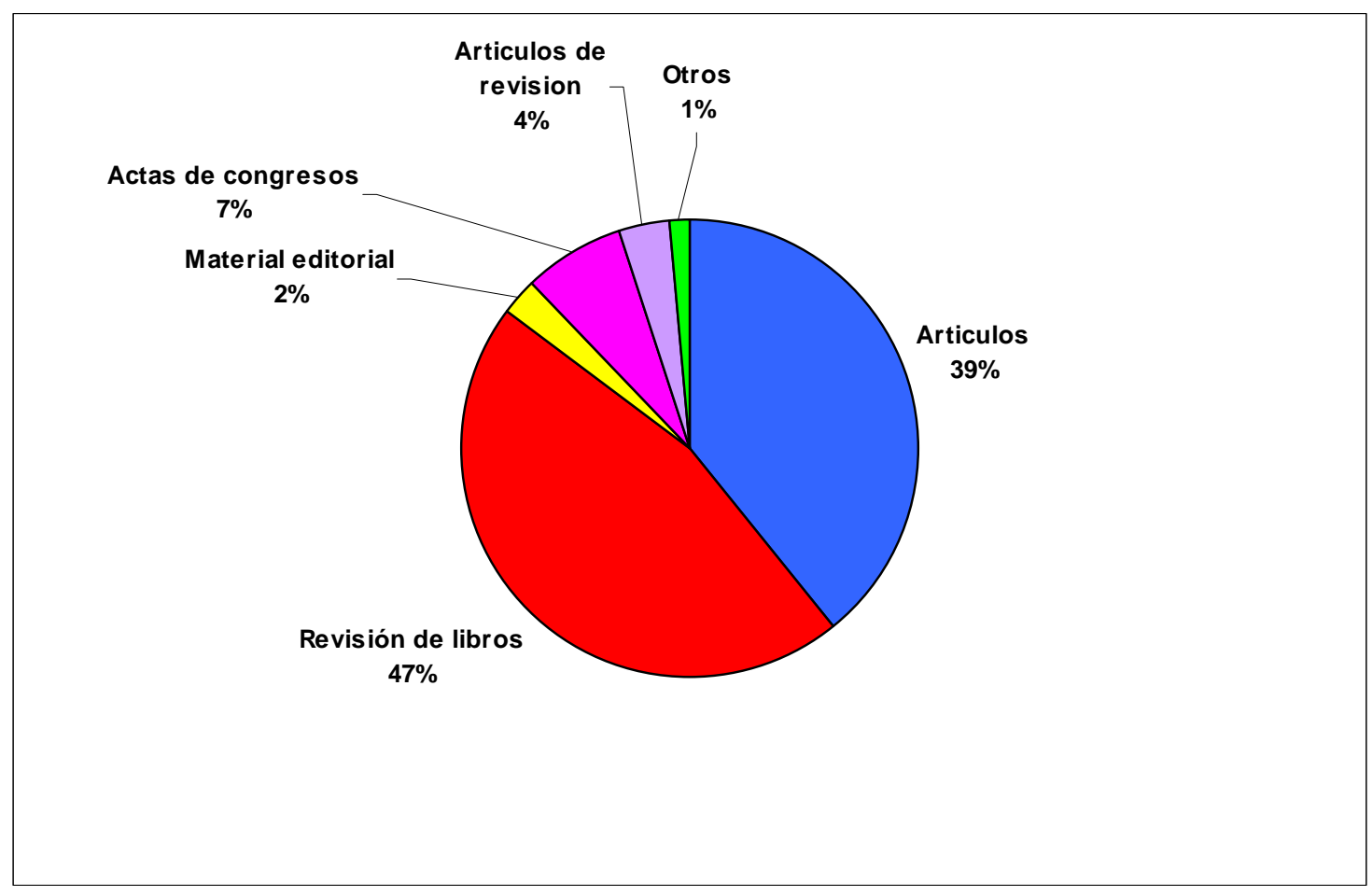

Figura 2. Distribución de la producción científica española por tipología documental. Fuente: Elaboración propia, a partir de la base de datos SCI y SSCI

\section{Los idiomas}

Como se puede ver en la Figura 3, las investigaciones españolas en historia de la ciencia, en revistas internacionales, se publican mayoritariamente en inglés (76'4\%), siendo el español y el francés, los idiomas utilizados en segundo y tercer lugar. Se observa un incremento en la utilización del idioma español a lo largo del periodo analizado, como consecuencia de la incorporación de revistas españolas en las bases de datos del ISI en los últimos años analizados².

\footnotetext{
${ }^{2}$ A partir del año 2006 se incluyen los trabajos publicados en la revista Dynamis y desde el 2008 los de la revista Asclepio.
} 


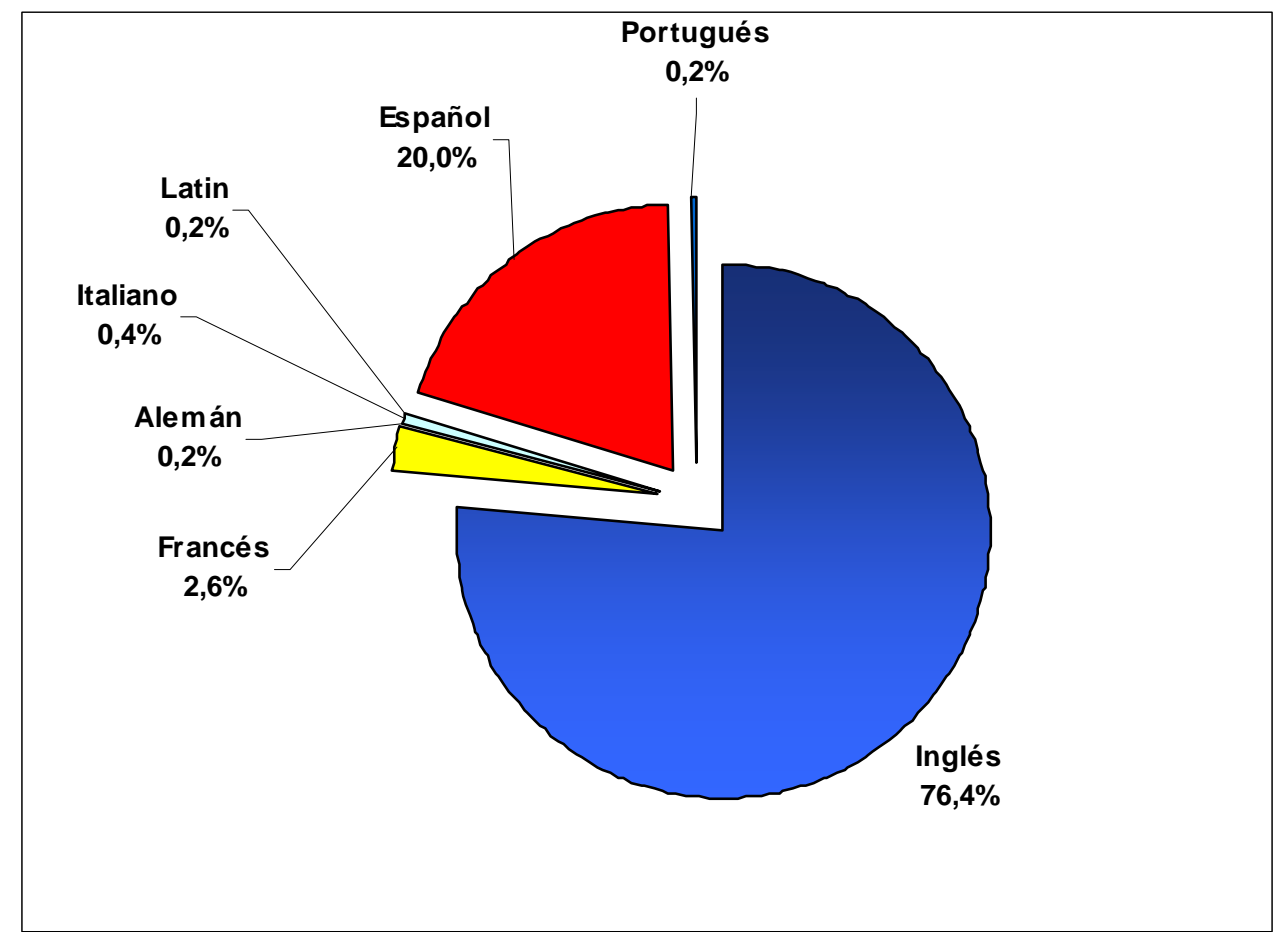

Figura 3. Distribución de la producción científica española por idioma de publicación. Fuente: Elaboración propia, a partir de la base de datos $\mathrm{SCl}$ y $\mathrm{SSCl}$

\section{Las revistas científicas}

El número total de revistas incluidas en la categoría History \& Philosophy of Science recogidos por las bases de datos JCR ( $\mathrm{SCl}$ y $\mathrm{SSCl}$ ) durante el período 1990-2009 es de 51. Un aspecto que llama la atención es que el número de revistas recogidas en la categoría HPS de la base de datos JCR del $\mathrm{SCl}$ es superior al de las recogidas en la categoría HPS de la base de datos J CR del SSCI. A lo largo del periodo estudiado, el número total de revistas recogidas en la base de datos JCR del SCl han sido 44, mientras que en la JCR del SSCI han sido 33. También se ha observado que hay 24 revistas que están recogidas simultáneamente en las dos bases de datos.

\section{Dispersión de la literatura científica}

Los 548 trabajos publicados en revistas clasificadas en la categoría HPS del JCR ( $\mathrm{SCl}$ y $\mathrm{SSCl}$ ), se han publicado en un total de 43 revistas. Existen 8 revistas, en las que no se ha publicado ningún trabajo realizado en alguna institución española (ver anexo I). También, tal como se muestra en la tabla 1, no todas las revistas han sido igualmente utilizadas por los investigadores. La revista donde se han publicado un mayor número de trabajos ha sido en la revista española Dynamis, que junto con la segunda revista en el ranking, Isis, acumulan más del $27 \%$ de toda la producción científica española en historia de la ciencia durante el período estudiado. 
Título de las revistas

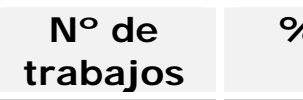

\begin{tabular}{|c|c|c|c|}
\hline 1 & Agricultural History & 6 & 1,05 \\
\hline 2 & Agriculture and Human Values & 1 & 0,18 \\
\hline 3 & American J ournal of Bioethics & 6 & 1,05 \\
\hline 4 & Annals of Science & 19 & 3,34 \\
\hline 5 & Archive for History of Exact Sciences & 20 & 3,51 \\
\hline 6 & $\begin{array}{l}\text { Asclepio-Revista de Historia de la Medicina y de la } \\
\text { Ciencia }\end{array}$ & 21 & 3,69 \\
\hline 7 & Biology \& Philosophy & 11 & 1,93 \\
\hline 8 & British J ournal for The History of Science & 14 & 2,46 \\
\hline 9 & British J ournal for The Philosophy of Science & 10 & 1,76 \\
\hline 10 & Bulletin of The History of Medicine & 11 & 1,93 \\
\hline 11 & Cryptologia & 2 & 0,35 \\
\hline 12 & Dynamis & 98 & 17,22 \\
\hline 13 & Endeavour & 3 & 0,53 \\
\hline 14 & Epistemologia & 1 & 0,18 \\
\hline 15 & Historia Mathematica & 19 & 3,34 \\
\hline 16 & $\begin{array}{l}\text { Historical Studies in the Physical And Biological } \\
\text { Sciences }\end{array}$ & 1 & 0,18 \\
\hline 17 & History And Philosophy of Logic & 45 & 7,91 \\
\hline 18 & History And Philosophy of The Life Sciences & 37 & 6,50 \\
\hline 19 & History of Science & 1 & 0,18 \\
\hline 20 & History of The Human Sciences & 2 & 0,35 \\
\hline 21 & Isis & 56 & 9,84 \\
\hline 22 & Journal for The History of Astronomy & 20 & 3,51 \\
\hline 23 & J ournal of Agricultural \& Environmental Ethics & 3 & 0,53 \\
\hline 24 & J ournal of The History of Biology & 5 & 0,88 \\
\hline 25 & Journal of The History of The Neurosciences & 4 & 0,70 \\
\hline 26 & Medical History & 18 & 3,16 \\
\hline 27 & Minerva & 5 & 0,88 \\
\hline 28 & Notes And Records of The Royal Society & 3 & 0,53 \\
\hline 29 & Nuncius-J ournal of The History of Science & 6 & 1,05 \\
\hline 30 & Osiris & 4 & 0,70 \\
\hline 31 & Perspectives in Biology and Medicine & 1 & 0,18 \\
\hline 32 & Philosophy of Science & 14 & 2,46 \\
\hline 33 & Physics In Perspective & 1 & 0,18 \\
\hline 34 & Public Understanding of Science & 11 & 1,93 \\
\hline 35 & Revista Mexicana De física E & 4 & 0,70 \\
\hline 36 & Science And Engineering Ethics & 7 & 1,23 \\
\hline 37 & Science in Context & 6 & 1,05 \\
\hline 38 & Social History of Medicine & 12 & 2,11 \\
\hline 39 & Social Studies of Science & 5 & 0,88 \\
\hline 40 & Studies in History and Philosophy of Modern Physics & 12 & 2,11 \\
\hline
\end{tabular}




\begin{tabular}{|l|l|c|c|}
\hline 41 & Studies in History and Philosophy of Science & 16 & 2,81 \\
\hline 42 & Synthese & 25 & 4,39 \\
\hline 43 & Technology and Culture & 3 & 0,53 \\
\hline & Total trabajos & $\mathbf{5 6 9}$ & 100,00 \\
\hline
\end{tabular}

Tabla 1. Relación alfabética de revistas y número de trabajos (1990-2009)

Fuente: Elaboración propia, a partir de la base de datos SCl y SSCI (1990-2009)

En la tabla 2 se muestra, por orden decreciente, la relación de revistas donde se han publicado los trabajos y los años de publicación. Para facilitar la lectura, se ha agrupado el número de trabajos por quinquenios, de esta forma, se puede ver con mayor claridad, la evolución del número de trabajos publicados en cada una de las revistas a lo largo del tiempo y conocer que revistas son las utilizadas, con una mayor regularidad, por los investigadores españoles para difundir sus trabajos. Se observa que la revista utilizada con mayor regularidad y frecuencia durante el período estudiado es la revista americana Isis. Sin embargo, tal como se puede ver en la tabla, la revista española Dynamis es la que publica un mayor número de trabajos realizados por investigadores españoles ${ }^{3}$ durante el período analizado y, posiblemente, en los próximos años, la revista Dynamis junto con otra revista española, Asclepio ${ }^{4}$, sean las dos publicaciones que terminen liderando el ranking de las revistas que difundan un mayor número de trabajos sobre historia de la ciencia realizados por investigadores españoles.

\footnotetext{
${ }^{3}$ Los trabajos publicados en la revista Dynamis se indizan en las base de datos del ISI Web of Knowdledge desde el año 2006.

${ }^{4}$ Incluida en las bases de datos del ISI Web of Knowdledge desde el año 2008.
} 


\begin{tabular}{|c|c|c|c|c|c|c|}
\hline Rank & Títulos de las revistas & $\begin{array}{l}1995- \\
1999\end{array}$ & $\begin{array}{l}1990- \\
1994\end{array}$ & $\begin{array}{l}2000- \\
2004\end{array}$ & $\begin{array}{l}2005- \\
2009\end{array}$ & Total \\
\hline 1 & Dynamis & & & & 98 & 98 \\
\hline 2 & Isis & 15 & 9 & 19 & 13 & 56 \\
\hline 3 & History And Philosophy of Logic & 14 & 8 & 11 & 12 & 45 \\
\hline 4 & $\begin{array}{l}\text { History And Philosophy of The } \\
\text { Life Sciences }\end{array}$ & 21 & 3 & 7 & 6 & 37 \\
\hline 5 & Synthese & 2 & 2 & 10 & 11 & 25 \\
\hline 6 & $\begin{array}{l}\text { Asclepio-Revista de Historia de } \\
\text { la Medicina y de la Ciencia }\end{array}$ & & & & 21 & 21 \\
\hline 7 & $\begin{array}{l}\text { Archive for History of Exact } \\
\text { Sciences }\end{array}$ & 5 & 7 & 4 & 4 & 20 \\
\hline 7 & $\begin{array}{l}\text { Journal for The History of } \\
\text { Astronomy }\end{array}$ & & & 1 & 19 & 20 \\
\hline 8 & Annals of Science & 3 & 5 & 7 & 4 & 19 \\
\hline 8 & Historia Mathematica & 2 & 4 & 3 & 10 & 19 \\
\hline 9 & Medical History & 2 & & 4 & 12 & 18 \\
\hline 10 & $\begin{array}{l}\text { Studies in History and } \\
\text { Philosophy of Science }\end{array}$ & 5 & 4 & 3 & 4 & 16 \\
\hline 11 & $\begin{array}{l}\text { British Journal for The History of } \\
\text { Science }\end{array}$ & 3 & 5 & 3 & 3 & 14 \\
\hline 11 & Philosophy of Science & 2 & 1 & 7 & 4 & 14 \\
\hline 12 & Social History of Medicine & 7 & & 3 & 2 & 12 \\
\hline 12 & $\begin{array}{l}\text { Studies in History and } \\
\text { Philosophy of Modern Physics }\end{array}$ & & & 2 & 10 & 12 \\
\hline 13 & Biology \& Philosophy & 2 & 1 & 4 & 4 & 11 \\
\hline 13 & $\begin{array}{l}\text { Bulletin of The History of } \\
\text { Medicine }\end{array}$ & & & 4 & 7 & 11 \\
\hline 13 & Public Understanding of Science & 2 & & 4 & 5 & 11 \\
\hline 14 & $\begin{array}{l}\text { British Journal for The } \\
\text { Philosophy of Science }\end{array}$ & 2 & 2 & 3 & 3 & 10 \\
\hline 15 & Science And Engineering Ethics & & & 2 & 5 & 7 \\
\hline 16 & Agricultural History & & 1 & & 5 & 6 \\
\hline 16 & American Journal of Bioethics & & & & 6 & 6 \\
\hline 16 & $\begin{array}{l}\text { Nuncius-Journal of The History } \\
\text { of Science }\end{array}$ & & & & 6 & 6 \\
\hline 16 & Science in Context & 2 & & 4 & & 6 \\
\hline 17 & Social Studies of Science & 2 & 1 & 1 & 1 & 5 \\
\hline 17 & Journal of The History of Biology & 1 & & 1 & 3 & 5 \\
\hline 17 & Minerva & 1 & & & 4 & 5 \\
\hline 18 & $\begin{array}{l}\text { Journal of The History of The } \\
\text { Neurosciences }\end{array}$ & & & & 4 & 4 \\
\hline 18 & Osiris & & 1 & 3 & & 4 \\
\hline 18 & Revista Mexicana de fisica $\mathrm{E}$ & & & & 4 & 4 \\
\hline 19 & Endeavour & 2 & 1 & & & 3 \\
\hline 19 & $\begin{array}{l}\text { Journal of Agricultural \& } \\
\text { Environmental Ethics }\end{array}$ & & & & 3 & 3 \\
\hline 19 & Notes and Records of The & & & & 3 & 3 \\
\hline
\end{tabular}




\begin{tabular}{|c|c|c|c|c|c|c|}
\hline & Royal Society & & & & & \\
\hline 19 & Technology and Culture & & & 1 & 2 & 3 \\
\hline 20 & Cryptologia & & & & 2 & 2 \\
\hline 20 & History of The Human Sciences & & 1 & & 1 & 2 \\
\hline 21 & Agriculture and Human Values & & & & 1 & 1 \\
\hline 21 & Epistemología & & & & 1 & 1 \\
\hline 21 & $\begin{array}{l}\text { Historical Studies in the Physical } \\
\text { And Biological Sciences }\end{array}$ & & & 1 & & 1 \\
\hline 21 & History of Science & & & & 1 & 1 \\
\hline 21 & $\begin{array}{l}\text { Perspectives in Biology and } \\
\text { Medicine }\end{array}$ & 1 & & & & 1 \\
\hline \multirow[t]{2}{*}{21} & Physics In Perspective & & & & 1 & 1 \\
\hline & Total trabajos & 96 & 56 & 112 & 305 & 569 \\
\hline
\end{tabular}

Tabla 2. Distribución de las revistas por número de trabajos y quinquenios

\section{Los autores}

De los 421 autores que han participado en la realización de los trabajos analizados, independientemente del tipo documental, se observa que la distribución de su productividad es muy distinta. Existen 307 autores (72'92\%) durante el período analizado, que han publicado un solo trabajo, 107 autores (25'42\%) que han colaborado en la realización entre 2 y 9 trabajos y 7 autores (1'66\%) que son grandes productores, ya que han colaborado en la realización de 10 o más trabajos durante el período analizado. De entre este conjunto de autores con gran producción destacan Antoni Malet Tomás (23 trabajos), Esteban Rodríguez Ocaña (23 trabajos) y Jon Pérez Laraudogoitia (15 trabajos).

Sin embargo, cuando se analiza el número de autores, que han colaborado en la publicación de artículos originales o de revisión, se observa que el número de autores disminuye considerablemente, siendo en este caso, solamente son 311 los autores que han publicado, durante el período analizado, algún artículo original o de revisión. Entre todos estos autores, se observa que son 254 (81'67\%) los que han publicado un solo artículo, $54\left(17^{\prime} 36 \%\right)$ los que han publicado entre 2 y 5 artículos y solamente $3\left(0^{\prime} 96 \%\right)$, los autores que han publicado 6 o más artículos. En este conjunto de grandes productores destacan Jon Pérez Laraudogoitia (12 artículos), Miguel Ángel Granada ( 7 artículos) y J osé Luis Mancha (6 artículos). 


\section{Colaboración de los autores}

La relación de coautoría en los artículos publicados en revistas científicas permite analizar en alguna medida la estructura de colaboración dentro de una comunidad académica. En este trabajo, con la información obtenida de los autores se ha construido la red de productividad y coautorías de los autores que han publicado algún trabajo en las revistas clasificadas bajo la materia HPS del JCR ( $\mathrm{SCl}$ y SSCI).

Se ha respetado el formato de los nombres de los autores tal y como aparece en las dos bases de datos consultadas, sin embargo, se ha procedido a la normalización del nombre de aquellos autores que tienen diferentes formatos en la base de datos. Para estos casos, se ha normalizado la información utilizando el formato que se ha considerado más fácil para la identificación de autor, como es el de los dos apellidos del autor unidos por un guión seguido de las iniciales del nombre. De esta forma, en los casos como Ron, JMS y Sánchez-Ron, JM se ha seleccionado la segunda opción, al igual que en los casos en los que aparecen los dos apellidos unidos sin guión o con guión también se ha normalizado el nombre de los autores, utilizando la segunda opción.

Se observa que aunque en muchas áreas científicas, la ciencia de nuestros días se hace en colaboración, en los trabajos realizados por investigadores del área de historia de la ciencia, que trabajan en nuestro país, observamos que existe poca colaboración. El $81,39 \%$ de los trabajos han sido firmados por un solo autor y solamente el $18,61 \%$ de los trabajos han sido realizados en colaboración entre dos o más autores.

En la Figura 4 se muestran las relaciones de colaboración científica entre los autores que trabajan en algún institución española y que han publicado algún trabajo en las fuentes de información consultadas. Los círculos representan los autores y las líneas indican las colaboraciones entre ellos. El grosor de los círculos indica el mayor o menor número de trabajos que ha realizado un autor. Existen 96 grupos de colaboración, siendo el más frecuente el formado por la colaboración entre dos autores (46 grupos), seguido del formado por tres autores (22 grupos). Llama la atención que existen dos grupos de colaboración de gran tamaño, formado cada uno de ellos con más de 20 autores. Hay que tener en cuenta que en esta red estamos representando aquellos autores que han publicado algún trabajo en colaboración, independientemente del tipo documental y, en estos casos, se observa que el grupo más numeroso, está formado por 23 autores que han colaborado en la realización del artículo titulado "The Enscot: The European network of science communication teachers", que fue publicado en el año 2003 en 
la revista Public Understandig of Science. En el caso del segundo grupo con mayor número de autores, se trata de una editorial publicada en la revista Science and Engineering Meeting, en el año 2006 y que se titula "Summary: The Budapest Meeting 2005 Intensified Networking on Ethics of Science - The case of reproductive cloning, germline gene therapy and human dignity" y que fue firmada por 22 autores.

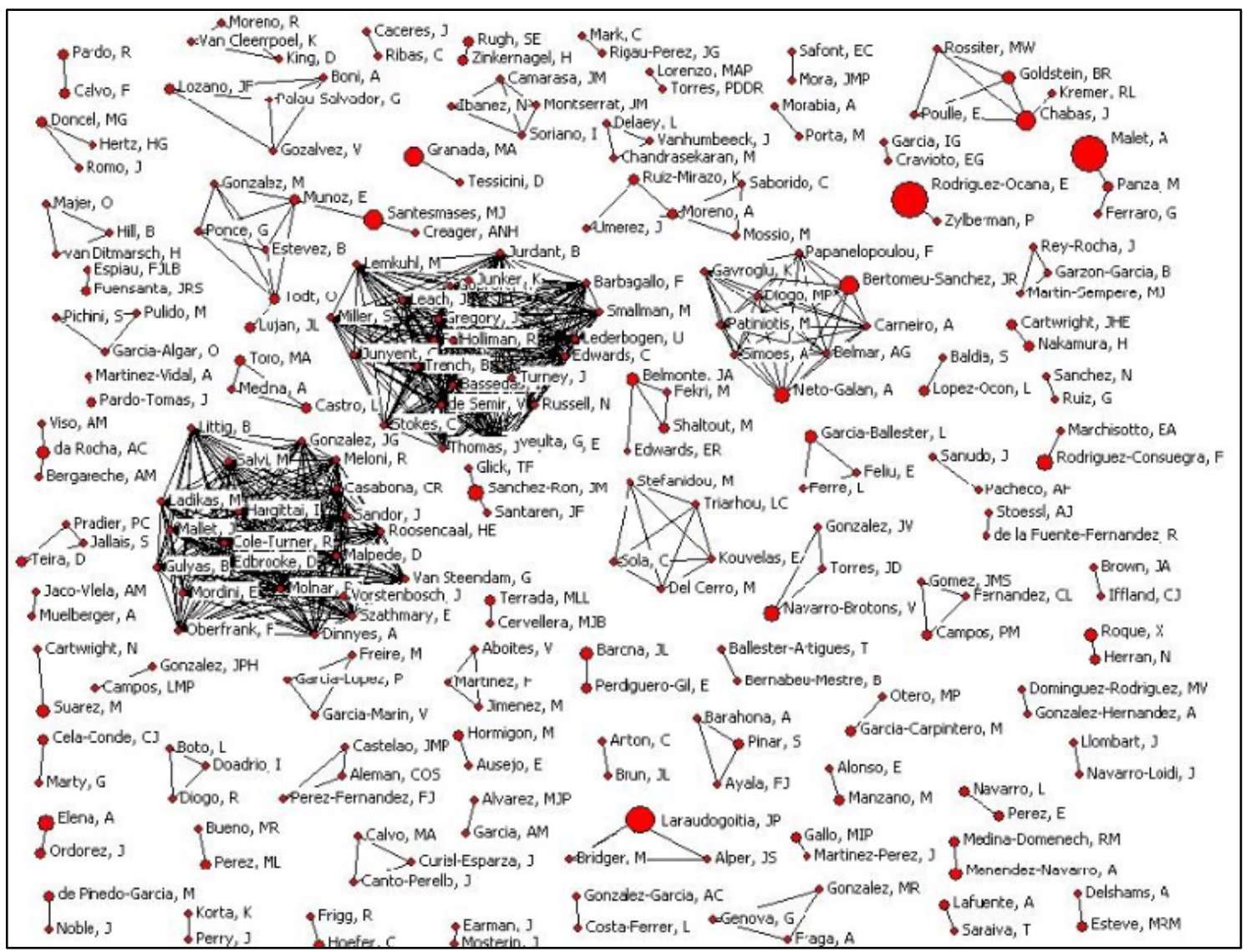

Figura 4. Red de colaboración científica entre los autores

Fuente: Elaboración propia, a partir de la base de datos SCl y SSCI (1990-2009)

En la Figura 5 se presenta una red con los autores de los artículos originales o de revisión. En este gráfico se han representado los autores que han publicado más de un trabajo durante el periodo analizado y también a los autores que han realizado algún trabajo en colaboración. En este caso, puede observarse que el valor más frecuente de firmas por artículo es de 2. El número de grupos de autores que han publicado algún artículo en coautoría es de 41, siendo el más frecuente el de formado por 2 autores ( 25 grupos) o 3 autores ( 11 grupos). El tamaño de los nodos viene dado, al igual que en el resto de los nodos incluidos en el gráfico, por la mayor o menor productividad del autor. En el gráfico se han representado con el color azul los nodos de los autores que, destacan, dentro de su grupo, por el papel 
de unión o intermediación que tienen entre los diferentes autores del grupo al que pertenecen.

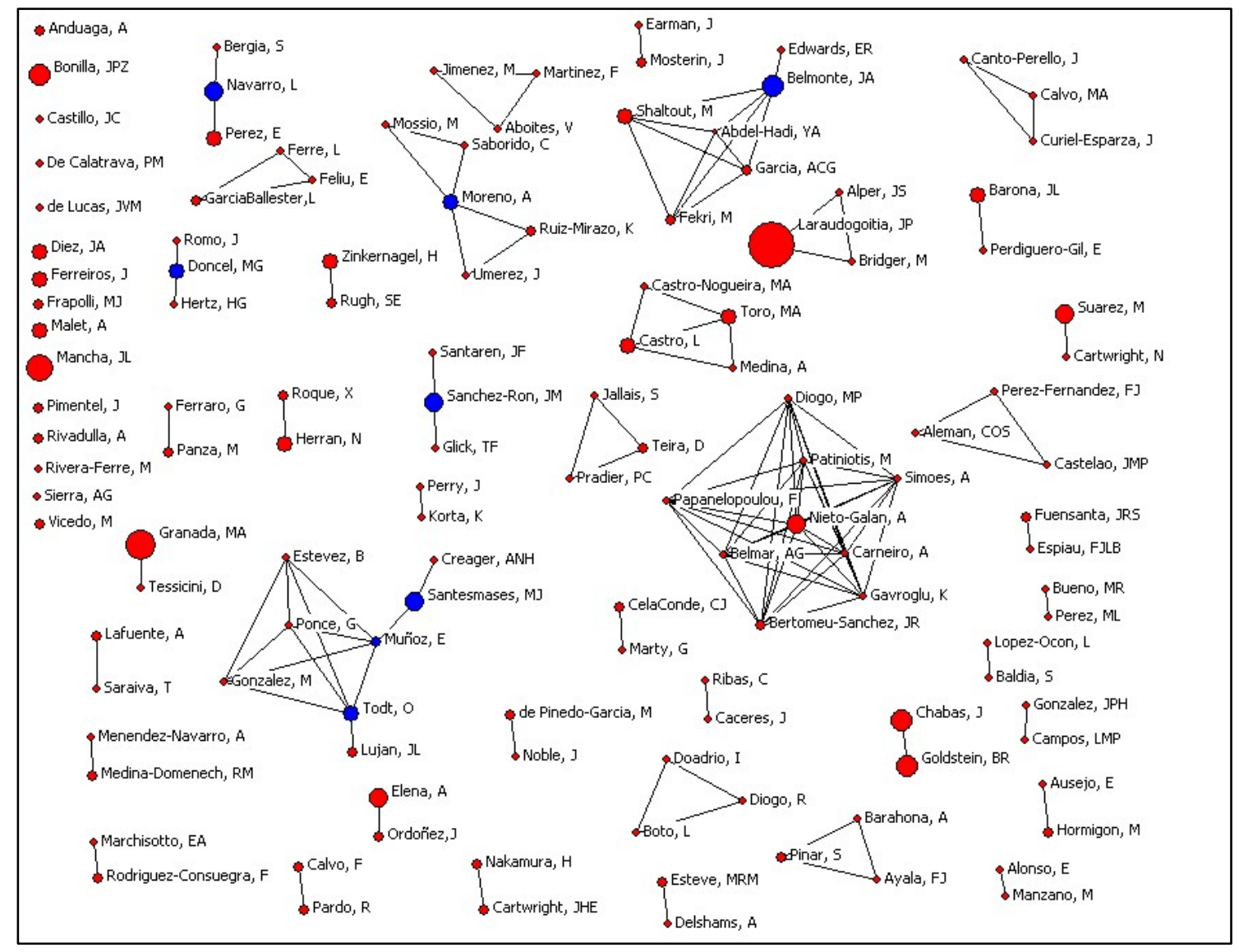

Figura 5. Red de autores de artículos originales y revisiones Fuente: Elaboración propia, a partir de la base de datos SCl y SSCI (1990-2009)

Por último, con el fin de poder conocer con mayor precisión, los hábitos de publicación y colaboración de los autores, se ha representado una red solamente con aquellos autores que han publicado, durante el período estudiado, dos o más artículos (Figura 6). Llama la atención que, al contrario de lo que sucede en otras disciplinas científicas, donde los mayores productores son los que suelen tener un mayor número de trabajos realizados en colaboración, sin embargo, en este caso, predominan los nodos aislados, es decir, existe un gran número de autores que realizan su trabajo sin colaboración, incluso los grandes productores. Tal como se observa en el gráfico, los grupos más frecuentes son los formados por dos autores. En este gráfico, el tamaño del nodo indica el mayor o menor número de artículos realizados por un autor y el grosor de la línea indica el mayor o menor número realizado en colaboración con otros autores. 


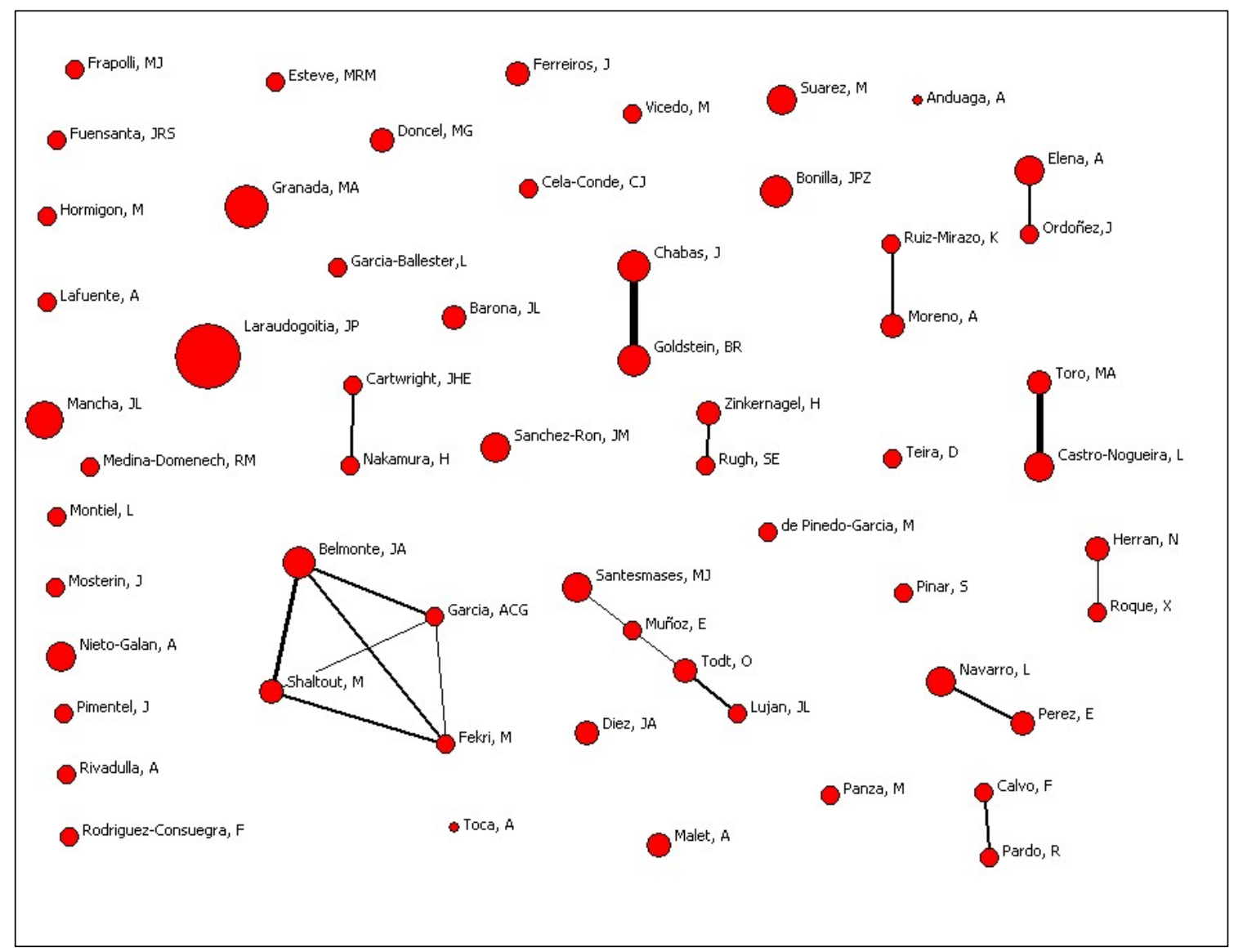

Figura 6. Red de autores de 2 o más artículos

Fuente: Elaboración propia, a partir de la base de datos SCl y SSCl (1990-2009)

\section{Conclusiones y perspectivas}

Este análisis preliminar sobre la actividad científica española en historia de la ciencia, se ha centrado en la obtención de indicadores bibliométricos a partir de los datos de una selección de bases de datos, que recogen las revistas científicas con mayor difusión internacional. Los resultados que aquí se muestran, hay que tomarlos con cierta cautela, dado que este análisis preliminar se ha centrado en la obtención de datos bibliométricos a partir de una sola categoría temática, History \& Philosophy of Science (HPS), utilizada por dos bases de datos (JCR del SCl y JCR del SSCI).

La historia de la ciencia, desde el punto de vista documental, es una disciplina compleja, porque por su multidisciplinariedad y por los hábitos de publicación de sus propios investigadores existe una gran dispersión en sus publicaciones. Los investigadores de esta disciplina utilizan una gran variedad de soportes documentales para la publicación de sus trabajos, destacando el papel relevante que tienen las monografías. Pero además, también hay que tener en cuenta que la 
multidisciplinareidad de la propia disciplina trae como consecuencia que el número de revistas científicas que utilizan los investigadores para publicar sus trabajos, sea muy amplio.

Con este trabajo se ha realizado una primera aproximación a la producción científica de los últimos veinte años en revistas internacionales de su especialidad, que nos ha permitido conocer los autores más relevantes y las revistas internacionales más utilizadas. Sin embargo, futuras líneas de trabajo deberán incidir en analizar los trabajos publicados por los investigadores de esta disciplina en revistas científicas internacionales de otras categorías temáticas, así como en revistas científicas de difusión nacional.

Asimismo, se podría realizar un estudio más exhaustivo de la disciplina, caracterizando los ámbitos temáticos de investigación de los grupos identificados, que podría revelar aspectos interesantes sobre los hábitos de publicación y las diferentes líneas de investigación. La realización de este tipo de análisis aportaría a la disciplina un mayor conocimiento de la misma y el conocimiento de las características de la historia de la ciencia en España.

Hay que destacar, que la utilización complementaria de las técnicas bibliométricas y el análisis de redes se ha desvelado como una combinación mucho más útil de lo que se podría esperar de la simple suma de dos métodos distintos. Los gráficos de redes permiten objetivar grupos de trabajo que de otro modo sería difícil descubrir.

Un resultado llamativo del análisis realizado es la escasa colaboración existente entre los investigadores. Los trabajos con un solo autor siguen siendo la norma general en el caso de la historia de la ciencia, lo mismo que sucede en otras disciplinas pertenecientes a las áreas de ciencias sociales y, sobre todo, en las humanidades. También se observa que el número de trabajos realizados en colaboración, suele ser ocasional y es más frecuente en el caso de trabajos presentados en congresos o cartas al editor, sin embargo, puede considerarse como excepcional cuando se trata de publicar artículos originales o de revisión, ya que en estos casos, el número de trabajos realizados en colaboración disminuye y no existen apenas grupos consolidados.

Por último, hay que resaltar también el papel tan relevante que tienen las revistas en la difusión internacional de la investigación. Hay que destacar que el 20’91\% de toda la producción científica española, a nivel internacional, de historia de la ciencia, de los últimos veinte años, recogida por las bases de datos del ISI, ha sido publicada en dos revistas españolas, Dynamis y Asclepio. Revista de Historia 
de la Medicina y de la Ciencia, cuyos trabajos están siendo indizados en las bases de datos del ISI desde los años 2006 y 2008 respectivamente.

\section{Bibliografía}

Alfonso, F.; Bermejo, J.; Segovia, J. (2005). Impactología, impactitos, impactoterapia. Revista Española de Cardiología, 58: 1239-1245.

Camí, J. (1997). Impactología: diagnóstico y tratamiento. Medicina Clínica, 109: 515-524.

Cuenca, M. J. (2003). Humanidades, ciencias sociales y económicas en el plan nacional 2004-2007. Economía Industrial, 354, VI, 125-134.

Delgado López-Cozar, E.; Torres Salinas, D.; Jiménez Contreras, E.; Ruiz Pérez, R. (2006). Análisis bibliométrico y de redes sociales aplicado a las tesis bibliométricas defendidas en España (1976-2002): temas, escuelas científicas y redes académicas. Revista Española de Documentación Científica, 29: 493-524.

Fundación Española para la Ciencia y la Tecnología (2006). Libro blanco de la investigación en humanidades. Madrid: Fecyt, 14-14.

López Baena, A.J.; Valcárcel Cases, M.; Barbancho Medina, M. (2005). Propuesta de un sistema de evaluación de revistas científicas en las áreas de ciencias humanas y sociales. Revista Española de Documentación Científica, 28, 1, 222-248.

López Piñero, J.M. (1972). El análisis estadístico y sociométrico de la literatura científica. Valencia: Centro de Documentación e Informática Médica de la Facultad de Medicina.

López Piñero, J.M.; Terrada, M.L. (1992). Los indicadores bibliométricos y la evaluación de la actividad médico-científica. (I). Usos y abusos de la bibliometría. Medicina Clínica, 98: 64-68.

Machlup, F. (1962). The production and distribution of knowledge in the United States. Princeton: University Press.

Molina, J. L. (2001). El análisis de redes sociales. Una introducción. Barcelona: Edicions Bellaterra.

Molina, J.L., Muñoz, J. M., Domenech, M. (2002). Redes de publicaciones científicas: un análisis de la estructura de coautorías. Redes. Revista Hispana para el análisis de redes sociales, Vol. 1, \#3.

Olmeda-Gómez, C.; Perianes-Rodriguez, A., Ovalle-Perandones, M.A., GuerreroBote, V.P., Moya Anegón, F. de (2009). Visualization of scientific co-authorship in Spanish universities. From regionalization to internationalization. Aslib Proceedings, vol. 61, 1: 83-100.

Osca-Lluch, J.; Castro Martínez, E.; Fernández de Lucio, I.; Serra Sister, P. (2003). La producción científico-técnica de la Comunidad Valenciana. Revista d'Estudis Autònomics, 38: 193-303.

Osca-Lluch, J.; Velasco, E.; López, M.; Haba, J. (2009). Co-authorship and citation networks in Spanish history of science research. Scientometrics, 80: 375-385.

Russell, J.M., Ainsworth, S.; Narváez-Berthelemot, N. (2006). Colaboración científica de la Universidad Nacional Autónoma de México (UNAM) y su política institucional. Revista Española de Documentación Científica, vol. 29, 1: 56-73. 
Russell, J.M.; Ainsworth, S.; Del Rio, A.; Narváez-Berthelemot, N.; Cortés, H.D. (2007). Colaboración científica entre países de la región latinoamericana. Revista Española de Documentación Científica, vol. 30. 2: 180-198.

Russell, J.M.; Madera Jaramillo, M.J.; Ainsworth, S. (2009). El análisis de redes en el estudio de la colaboración científica. Redes. Revista Hispana para el análisis de redes sociales, Vol. 17, \#1: 39-47.

Terrada, M.L.; López Piñero, J.M.; Osca Lluch, J.; Almero, A. (1993). El libro médico español en los años ochenta. Estudio bibliométrico. Valencia: Instituto de Estudios Documentales e Históricos sobre la Ciencia.

Zulueta, M. A.; Bordons, M. (1999). La producción científica española en el área cardiovascular a través del Science Citation Index (1990-199). Revista Española de Cardiología, 52: 751-764. 


\section{ANEXO I}

Relación alfabética de las revistas clasificadas en la categoría History \& Philosophy of Science (HPS) de las bases de datos JCR ( $\mathrm{SCl}$ y $\mathrm{SSCl}$ ), que no han publicado trabajos realizados en instituciones españolas durante el período 1990-2009

\begin{tabular}{|l|l|l|}
\hline Título de la revista & País & \multicolumn{1}{c}{ BD } \\
\hline BERICHTE ZUR WISSENSCHAFTSGESCHICHTE & Alemania & $\mathrm{SCl}$ \\
\hline CONFIGURATIONS & Estados Unidos & $\mathrm{SSCl}$ \\
\hline HERALD OF THE RUSSIAN ACADEMY OF SCIENCES & Estados Unidos & $\mathrm{SCl}$ \\
\hline HISTORICAL STUDIES IN THE NATURAL SCIENCES & Estados Unidos $\mathrm{SCl}, \mathrm{SSCl}$ \\
\hline HYLE & Alemania & $\mathrm{SCl}, \mathrm{SSCl}$ \\
\hline IEEE ANNALS OF THE HISTORY OF COMPUTING & Estados Unidos $\mathrm{SCl}$ \\
\hline JOURNAL OF THE HISTORY OF MEDICINE AND ALLIED SCIENCES & Estados Unidos & $\mathrm{SCl}, \mathrm{SSCl}$ \\
\hline NURSING HISTORY REVIEW & Estados Unidos $\mathrm{SC}$
\end{tabular}

